Objectives Highly sensitive and specific assays for diagnosis of Neisseria gonorrhoeae (NG) are imperative. Unfortunately, several commercial and in house NG nucleic acid amplification tests (NAATs) have shown suboptimal specificity. The Neisseria gonorrhoeae PCR kit (GeneProof) is a novel NG dual-target (porA pseudogene and $16 \mathrm{~S}$ rRNA gene) real-time PCR. Herein, the analytical sensitivity and specificity of the NG PCR kit (GeneProof) were evaluated using a collection of well-characterised gonococcal isolates $(\mathrm{n}=105)$, with a global representativeness, and non-gonococcal Neisseria isolates ( $\mathrm{n}=149 ; 21$ different species and subspecies), as well as specimens positive with three other commercially available NAATs $(\mathrm{n}=37)$.

Methods DNA was extracted from all samples using the NorDiag Bullet robot (NorDiag ASA Company) and kept in $-20^{\circ} \mathrm{C}$ prior to testing. All samples were tested on LightCycler 2.0 (Roche Molecular Systems Inc.) by adding $10 \mu \mathrm{l}$ of DNA to $30 \mu \mathrm{l}$ NG PCR kit (GeneProof) reagent mix.

Results All 105 gonococcal isolates, including three porA mutants, were detected and none of the 149 non-gonococcal Neisseria strains were false positive. Accordingly, the assay displayed $100 \%$ analytical sensitivity and specificity. The analytical sensitivity was 1-10 genome copies per reaction. All positive samples from the Abbott RealTime PCR CT/NG (Abbott Laboratories) $(n=5)$ and COBAS 4800 (Roche Molecular Systems Inc.) systems $(n=8)$ were verified. However, for the BD ProbeTec ET/Ox Chlamydia trachomatis and Neisseria gonorhoeae Amplified DNA (Becton Dickinson) only eight out of 24 low-positive samples could be verified as true positive.

Conclusions This study shows that the GeneProof NG PCR kit is analytically highly specific and sensitive for detection of $N$. gonorrhoeae. This study also emphasises the importance of verifying $N$. gonorrhoeae NAAT positive specimens, particularly specimens that are low positive or from extragenital sites, with an alternative NAAT using a different target.

\section{P2.053 CHLAMYDIA TRACHOMATIS/NEISSERIA GONORRHOEAE SCREENING IN DUPLEX: SHOULD WE VERIFY N. GONORRHOEAE POSITIVE RESULTS?}

doi:10.1136/sextrans-2013-051184.0318

${ }^{1,2} \mathrm{C}$ Le Roy, ${ }^{1,2,30}$ Peuchant, ${ }^{4}$ Le Hen, ${ }^{4}$ B Bertrand-Salles, ${ }^{1,2,3} \mathrm{C}$ Bébéar,

1,2,3 B de Barbeyrac. 'University of Bordeaux, Bordeaux, France; '2Institut National de la Recherche Agronomique, Bordeaux, France; ${ }^{3}$ Centre Hospitalier Universitaire de Bordeaux, Bordeaux, France; " Maison Départementale de la Santé CDAG/CIDDIST, Bordeaux, France

The objective of this study was to assess the utility of a supplementary PCR test following a positive Abbott m2000 PCR test result for Neisseria gonorrhoeae (NG) issued from urogenital specimens tested in the Department of Bacteriology at the Bordeaux University Hospital, France in 2011-2013.

All NG-positive PCR specimens either negative with NG culture or without culture result, were retrieved and tested with two CT/ NG PCR tests: Cepheid GeneXpert CT/NG and Roche cobas 4800 $\mathrm{CT} / \mathrm{NG}$ (both targeting two genes). Analytical sensitivity of the three tests, Abbott, Cepheid and Roche for NG detection, was tested on serial dilutions (10-1 to 10-9) from one colony of NG suspended in cobas ${ }^{\circledR}$ PCR medium (Roche).

Of 11.010 specimens tested on Abbott m2000, the global prevalence of NG was $2.5 \%(277 / 11.010)$ and varied with the origin of the patient, from $0.8 \%$ at a free and anonymous STI screening centre (CDAG) to $6 \%$ at STI clinics (CIDDIST). Out of 215 specimens analysed, 112 were confirmed by culture or with a second PCR-positive sample, and 103 were tested with the two other PCR assays. A total of 197/215 NG-positive Abbott PCR results were confirmed. The overall positive predictive value (PPV) of the CT/NG Abbott test was $91.6 \%$, ranging from $73.5 \%$ for asymptomatic patients consulting at CDAG to $95.8 \%$ for symptomatic patients consulting at CIDDIST. Concern- ing the analytical sensitivity, the Cepheid test was 10 and 100 times more sensitive than the Abbott and the Roche tests, respectively.

In populations where the prevalence was $<1 \%$, the Abbott CT/ NG test had a PPV $<90 \%$ and therefore required confirmation testing. When NG screening is associated with that of CT in populations with variable prevalence, it should be recommended to either use a NG PCR test with two targets or confirm a positive result by another PCR test with a different target.

\section{P2.054 OPTIMAL PROCESSING OF CHLAMYDIA TRACHOMATIS SIMULATED SAMPLES FROM PROFICIENCY TESTING PANELS BY DILUTION WITH COBAS ${ }^{\odot}$ PCR MEDIA}

doi:10.1136/sextrans-2013-051184.0319

1J Engstrom-Melnyk, 'P Rodriguez, ${ }^{2} 0$ Liesenfeld, ${ }^{2}$ J Osiecki. 'Roche Diagnostics, Indianapolis, IN, United States; ${ }^{2}$ Roche Molecular Systems, Pleasanton, CA, United States

Background Proficiency materials are designed to resemble true clinical samples, yet challenges exist in procuring sufficient quantity of patient material. Simulated samples are often provided for this testing. Matrix effect with simulated samples can confound molecular assessment, having negative consequences for the laboratory through failed proficiency testing. This study was conducted to evaluate simulated urine samples provided for proficiency testing which generate invalid results with the cobas ${ }^{\circledR} \mathrm{CT} / \mathrm{NG}$ test.

Methods Simulated urine proficiency panels were acquired from a commercial proficiency testing provider. Panels were evaluated in triplicate by routine procedure at neat concentrations and processed with cobas $^{\odot}$ PCR media at the following dilutions 1:1, 1:5, 1:10, 1:20, 1:50,1:100,1:200, and 1:500. The samples were held at room temperature (1 complete set) for 1 hour prior to loading on the cobas $^{\odot} 4800$ system, while the second complete set of samples were processed 24 hours later. Samples were tested using two different cobas $^{\oplus} 4800$ workflows (400ul vs. 850ul of sample). Internal control and target $\mathrm{Ct}$ values were assessed for each sample to determine success of amplification.

Results Invalid results due to internal control failures were observed at neat concentrations of simulated urine samples. Incubation of samples for 1 hour or 24 hours in cobas ${ }^{\circledR}$ PCR media, showed no significant difference between target and IC Ct values indicating incubation period in cobas $^{\odot}$ PCR media does not impact performance. Simulated Urine Sample dilution of 1:5 in cobas ${ }^{\odot}$ PCR media using the 400ul sample input volume produced similar IC Ct values (mean $\mathrm{Ct}=35.5)$ to cobas $^{\odot} \mathrm{PCR}$ media tested alone (mean $\mathrm{Ct}=36$ ), and produced a robust target signal (mean $\mathrm{Ct}=22$ ).

Conclusions Proficiency testing materials may require optimization for use on commercially available systems. Optimal processing of simulated urine specimens can be achieved by dilution in cobas ${ }^{\odot}$ PCR media.

\section{P2.055 "P.I.D." OR ENDOMETRIOSIS? LAPAROSCOPIC ASSESSMENT, CHLAMYDIAL ANTIBODIES AND DYSMENORRHEA SYMPTOM SCORING IN WOMEN WITH ACUTE PELVIC PAIN}

doi:10.1136/sextrans-2013-051184.0320

1.2P Greenhouse, ${ }^{2} \mathrm{~V}$ Lees, ${ }^{3} \mathrm{C}$ Overton, ${ }^{4} \mathrm{~S}$ Vyas, ${ }^{4} \mathrm{~V}$ Akande, ${ }^{2,3} \mathrm{C}$ Bevan. ${ }^{1}$ Bristol Sexual Health Centre, Bristol, UK; ${ }^{2}$ Weston General Hospital, Weston-super-Mare, UK; ${ }^{3 B}$ Bistol Royal Infirmary, Bristol, UK; ${ }^{4}$ Southmead Hospital, Bristol, UK

Background Most women with endometriosis receive unnecessary antibiotics for "P.I.D." because both conditions present with pelvic pain and dyspareunia.

We used negative chlamydial antibody titre and laparoscopy to confirm diagnoses in women identified by dysmenorrhea symptom scoring (DSS) as more probably having endometriosis than PID. 